\title{
Differential expression of microRNA-675, microRNA-139-3p and microRNA-335 in benign and malignant adrenocortical tumours
}

\author{
K J Schmitz, ${ }^{1,2}$ J Helwig, ${ }^{1}$ S Bertram, ${ }^{1}$ S Y Sheu, ${ }^{1}$ A C Suttorp, ${ }^{1}$ J Seggewiß, ${ }^{3}$ \\ E Willscher, ${ }^{3}$ M K Walz, ${ }^{4} \mathrm{~K} \mathrm{Worm}^{1}{ }^{1} \mathrm{~K}$ W Schmid ${ }^{1}$
}

- A supplement is published online only. To view this file please visit the journal online (http://jcp.bmi.com).

${ }^{1}$ Institute of Pathology and Neuropathology, University Hospital of Essen, University of Duisburg-Essen, Essen, Germany

${ }^{2}$ Pathologisches Institut Recklinghausen, Recklinhausen, Germany

${ }_{3}^{3}$ Integrated Functional Genomics (IFG), Interdisciplinary Center for Clinical Research (IZKF),

University Hospital of Muenster, Westfalian Wilhelms-University of Muenster, Muenster, Germany

${ }^{4}$ Department of Surgery and Centre of Minimally Invasive Surgery, Kliniken Essen-Mitte, Essen, Germany

\section{Correspondence to}

Klaus J Schmitz, Institute of Pathology and Neuropathology, University Hospital Essen, University of Duisburg-Essen, Hufelandstrasse 55, Essen 45122, Germany;

schmitzies@versanet.de

Accepted 9 March 2011 Published Online First 6 April 2011

\begin{abstract}
Background For the clinical management of adrenocortical neoplasms it is crucial to correctly distinguish between benign and malignant tumours. Even histomorphologically based scoring systems do not allow precise separation in single lesions, thus novel parameters are desired which offer a more accurate differentiation. The tremendous potential of microRNAs (miRNAs) as diagnostic biomarkers in surgical pathology has recently been shown in a broad variety of tumours. Methods In order to elucidate the diagnostic impact of miRNA expression in adrenocortical neoplasms, a cohort of 20 adrenocortical specimens including normal adrenal tissue $(n=4)$, adrenocortical adenomas (ACAs) $(n=9)$, adrenocortical carcinomas (ACCs) $(n=4)$ and metastases $(n=3)$ was analysed using TaqMan low density arrays to identify specific miRNA profiles in order to distinguish between benign and malignant adrenocortical lesions. Results were validated in a validation cohort $(n=16)$. Results Concerning the differential diagnosis of ACAs and ACCs, 159 out of 667 miRNAs were up- and 89 were down-regulated in ACAs. Using real-time PCR analysis of three of the most significantly expressed single key miRNAs allowed separation of ACAs from ACCs. ACCs exhibited significantly lower levels of miR139-3p (up to 8.49-fold, $\mathrm{p}<0.001$ ), miR-675 (up to 23.25-fold, $p<0.001$ ) and miR-335 (up to 5.25-fold, $p<0.001)$. A validation cohort of 16 specimen with known Weiss score showed up-regulation of miR-335 and miR-675 in the majority of cases with probable malignant course, although overlapping values exist. Conclusion miRNA profiling of miR-675 and miR-335 helps in discriminating ACCs from ACAs. miRNA analysis may indicate malignant behaviour in cases with indeterminate malignant potential.
\end{abstract}

\section{INTRODUCTION}

MicroRNAs (miRNAs) are a class of recently discovered small RNA molecules that regulate the expression of genes at the translational level. ${ }^{12}$ The understanding of these tiny RNA molecules is rather limited, but studies indicate that miRNAmediated gene regulation is likely to play key roles in human development, cellular differentiation and oncogenesis. MiRNAs are endogenously expressed short non-coding RNAs with 18-25 nucleotides in length capable of repressing protein translation through binding to target messenger RNA (mRNA). The single stranded miRNAs, derived from miRNA duplex complexes, can associate with the RNA-induced silencing complex (RISC) ${ }^{3}$ and cause the translational repression or cleavage of these target mRNA sequences.

The discovery of the first miRNAs in 1993 immediately stimulated research in the medical field, and the question arose as to what extent miRNA analysis may be utilised in surgical pathology. Recent research indicated that evaluation of miRNA expression levels may substantially contribute to the understanding of cancer development, progression and treatment. ${ }^{4}$ Analysis of miRNA up- and down-regulation has been shown to be useful to support the diagnosis in a broad variety of lesions including, for example, endocrine tumours from the thyroid gland. ${ }^{5-10}$

Recently Giordano et al described a set of miRNAs related to prognosis in adrenocortical carcinoma (ACC). ${ }^{11}$ Whereas the discrimination of normal adrenal tissue and adrenocortical adenoma (ACA) is usually not challenging to the pathologist, the differential diagnosis of adrenocortical adenoma from ACC is notoriously difficult. ACC is a rare tumour which accounts for less than $0.2 \%$ of all human malignancies. Up to two-thirds of cases have distant metastases at the time of diagnosis, and the mortality exceeds $90 \%$ with a mean survival of less than 30 months. ${ }^{12}{ }^{13}$ Unlike in other malignancies, the diagnosis of ACC cannot be based on simple morphological parameters. To overcome the problem, distinct sets of histopathological criteria for the discrimination of benign and malignant adrenocortical neoplasm have been proposed. $^{12} 1415$ Nevertheless a clear separation of these neoplasms remains impossible unless the patient develops metastases. This is mainly due to the fact that such scores are based on histomorphological features that are likely to suffer from interobserver variation.

Little is currently known about the microRNA expression profile in adrenocortical tumours. In a very recent paper several miRNAs are described to be of diagnostic as well as prognostic value in ACAs and $\mathrm{ACCs},{ }^{16}$ indicating the potential value of microRNA expression patterns in diagnostic pathology. In order to further investigate the miRNA expression profiles in adrenal tissue, we performed miRNA analyses on a series of normal, benign and malignant adrenocortical tissue to analyse 667 human miRNA, followed by real-time PCR based validation of miRNA expression of single key miRNAs with potential diagnostic value in discriminating ACC from ACA. In a third step we compared miRNA expression levels of key miRNAs with the Weiss score in a validation cohort. online under the BMJ Journals unlocked scheme, see http:// jcp.bmi.com/site/about/ unlocked.xhtml 


\section{MATERIAL AND METHODS Patient material}

Formaldehyde-fixed paraffin-embedded tissue collection samples examined in the years from 2001 to 2008 were retrieved from the files of the Institute of Pathology and Neuropathology, University Hospital of Essen, University of Duisburg-Essen, German. All cases selected were operated and seen for follow-up at the Department of Surgery and Centre of Minimally Invasive Surgery, Kliniken Essen-Mitte, Essen, Germany and the Department of Transplantation and General Surgery, University Hospital Essen, Essen, Germany. The test cohort comprised normal adrenocortical tissue $(n=4)$ taken from nephrectomy specimens, ACAs associated with Cushing syndrome $(n=5)$ and associated with Conn syndrome $(n=4)$, ACCs $(n=4)$ and tissue from metastases of ACCs $(n=3)$. The validation cohort comprised 15 primary adrenocortical lesions and one lung metastasis, all scored according to the criteria of Weiss et al. ${ }^{15}$ The tumours for this study were reviewed by KJS. Tumour classification was performed according to the current WHO classification (2004). ${ }^{13}$ All ACC cases of the test study demonstrated at least three criteria associated with malignancy, for example necrosis, capsular invasion, vascular invasion, increased mitotic activity, significant atypia and/or metastases according the criteria proposed by Weiss et al. ${ }^{15}$ Table 1 summarises clinicopathological data of all ACCs.

Tissue was retrieved from paraffin-embedded material using a punch tool of $0.6 \mathrm{~mm}$ diameter. At least three complete cores were used for RNA extraction. Standard H\&E staining was performed in order to ensure that removed tissue samples were representative for the lesion.

\section{RNA preparation}

miRNA was prepared with the Recover All Total Nucleic Acid Isolation Kit (Applied Biosystems, Darmstadt, Germany) according to the kit protocol. In brief, tissue cylinders were deparaffinised by xylene-ethanol extraction and subsequently digested with proteinase $\mathrm{K}$. Nucleic acids were bound to the silica matrix of mini spin columns, washed, DNAse digested and finally eluted with a volume of $60 \mu$ l elution buffer.

\section{Reverse transcription}

miRNAs were reverse transcribed with the TaqMan miRNA Reverse Transcription Kit (Applied Biosystems, Darmstadt, Germany), using $60 \mathrm{ng}$ total RNA and pools of miRNA specific stemloop primers (Megaplex RT-Primers Pool A and B, Applied Biosystems). After reverse transcription, the cDNA was preamplified with Megaplex PreAmp Primers (Pool A and B) according to the recommendations of the supplier (Applied Biosystems).

\section{TaqMan low density arrays}

miRNA analyses were performed using TaqMan MicroRNA Array v2.0 (Applied Biosystems). Each sample was assayed with an A \& B card resulting in the analysis of a total of 667 miRNA. Each miRNA was measured in duplicate.

\section{Real-time PCR validation of single miRNAs}

Key miRNAs from TaqMan low density arrays (TLDAs) were selected for validation using real-time PCR. RNA from the previous TLDAs was used to quantify miRNA levels of hsa-miR335-4395296, hsa-miR-139-3p-4395424 and hsa-miR-675-4395. RNU48 served as endogenous control for normalisation. MiRNA levels were compared with that of the normal adrenocortical tissue. Quantitative reverse transcriptase PCR was done for each sample in triplicate. The $12 \mu \mathrm{l}$ real-time PCR mixture contained $5.25 \mu \mathrm{l}$ cDNA (diluted 1:25), TaqMan Universal PCR master mix (No AmpErase UNG) and $1 \mu \mathrm{l}$ of TaqMan assay hsa-mir-675/ hsa-miR-335/hsa-miR-139-3p. The reaction was imitated at $95^{\circ} \mathrm{C}$ for $10 \mathrm{~min}$, followed by 40 cycles of $95^{\circ} \mathrm{C}$ for $15 \mathrm{~s}$, and $60^{\circ} \mathrm{C}$ for $1 \mathrm{~min}$. The reaction was carried out using an Applied Biosystem 7500 fast real-time PCR system (Applied Biosystems).

\section{miRNA target prediction}

The DIANA-microT algorithm was used to predict possible target genes of differentially expressed miRNAs. ${ }^{17}$ MicroT identifies miRNA targets by combining TargetScan and PicTar predictions and has been reported to be the most selective algorithm in a comparative study. ${ }^{18}$

\section{Statistical analysis}

\section{TaqMan low density arrays}

Data obtained by the TLDAs was analysed using the StatMiner 3.0 software (Integromics, Madrid, Spain). Possible outliers in each biological group were identified by correlation heatmaps, and samples with a Pearson index $>0.85$ were discarded from further analyses. The imputation was performed using the aggregation method 'median' and the experimental factor 'biological group'. Detection thresholds were set to 35 and the best fitting endogenous control (RNU48-4373383 and MammU6-4395470) was calculated with the Normfinder algorithm. A parameter t-test (Limma) was applied for the relative quantification.

\section{Real-time PCR}

Default threshold settings were used to determine the threshold cycle data. The relative amount of each miRNA was calculated using the comparative threshold $(\mathrm{Ct})$ method with $\Delta \mathrm{Ct}=\mathrm{Ct}$ (miRNA)-Ct(RNU48). Relative quantification of miRNA expression was calculated with the $2^{-\Delta \Delta \mathrm{Ct}}$ method (Applied Biosystems User Bulletin No. 2 (P/N:4303859)). This method (called the comparative CT method) is widely used to present relative gene expression. ${ }^{19}$ Differences between miRNA expression levels among two groups were evaluated using a t-test; p-values $<0.05$ were considered significant. Statistics were performed with SPSS V.17.

\section{RESULTS \\ Test cohort \\ TLDA analysis}

For this study a microarray technique with 667 miRNAs was used to identify significantly $(\mathrm{p}<0.05)$ up- or down-regulated miRNAs

Table 1 Clinicopathological data of the four adrenocortical carcinomas in the test cohort

\begin{tabular}{|c|c|c|c|c|c|c|c|c|}
\hline $\begin{array}{l}\text { Adrenocortical } \\
\text { carcinomas } \\
\text { in test cohort }\end{array}$ & Size & Weight & $\begin{array}{l}\text { Vascular } \\
\text { invasion }\end{array}$ & $\begin{array}{l}\text { Capsular } \\
\text { invasion }\end{array}$ & $\begin{array}{l}\text { Significant } \\
\text { necrosis }\end{array}$ & $\begin{array}{l}\text { High nuclear } \\
\text { grade }\end{array}$ & $\begin{array}{l}\text { Increased mitotic } \\
\text { activity (>2 per } \\
10 \mathrm{HPF} \text { ) }\end{array}$ & Metastasis \\
\hline Case 1 & $14 \mathrm{~cm}$ & Not known & - & - & + & + & + & Not known \\
\hline Case 2 & $17 \mathrm{~cm}$ & $1078 \mathrm{~g}$ & - & - & + & + & + & Not known \\
\hline Case 3 & $18 \mathrm{~cm}$ & Not known & + & + & + & + & + & Yes \\
\hline Case 4 & $10 \mathrm{~cm}$ & Not Known & - & - & + & + & + & Yes \\
\hline
\end{tabular}

ACC, adrenocortical carcinoma; HPF, high power field. 
Figure 1 Unsupervised hierarchical cluster of microRNAs (miRNAs) and adrenocortical carcinoma (ACC) and adrenocortical adenoma (ACA) samples. Each row shows an miRNA, each column shows a sample. The miRNA tree is on the right, the sample tree is on top. The cluster shows miRNAs which separate ACCs from ACAs. The colour scale at the upper right illustrates the $\Delta \Delta$ Ct values of an miRNA across ACC and ACA samples: red for a negative $\Delta \Delta \mathrm{Ct}$ value and green for a positive $\Delta \Delta \mathrm{Ct}$ value. The clustering is done on $\Delta \Delta$ Ct values, which passed all filtering criteria across all samples. $C_{\text {, }}$ adrenocortical carcinoma; $A$, adrenocortical adenoma.

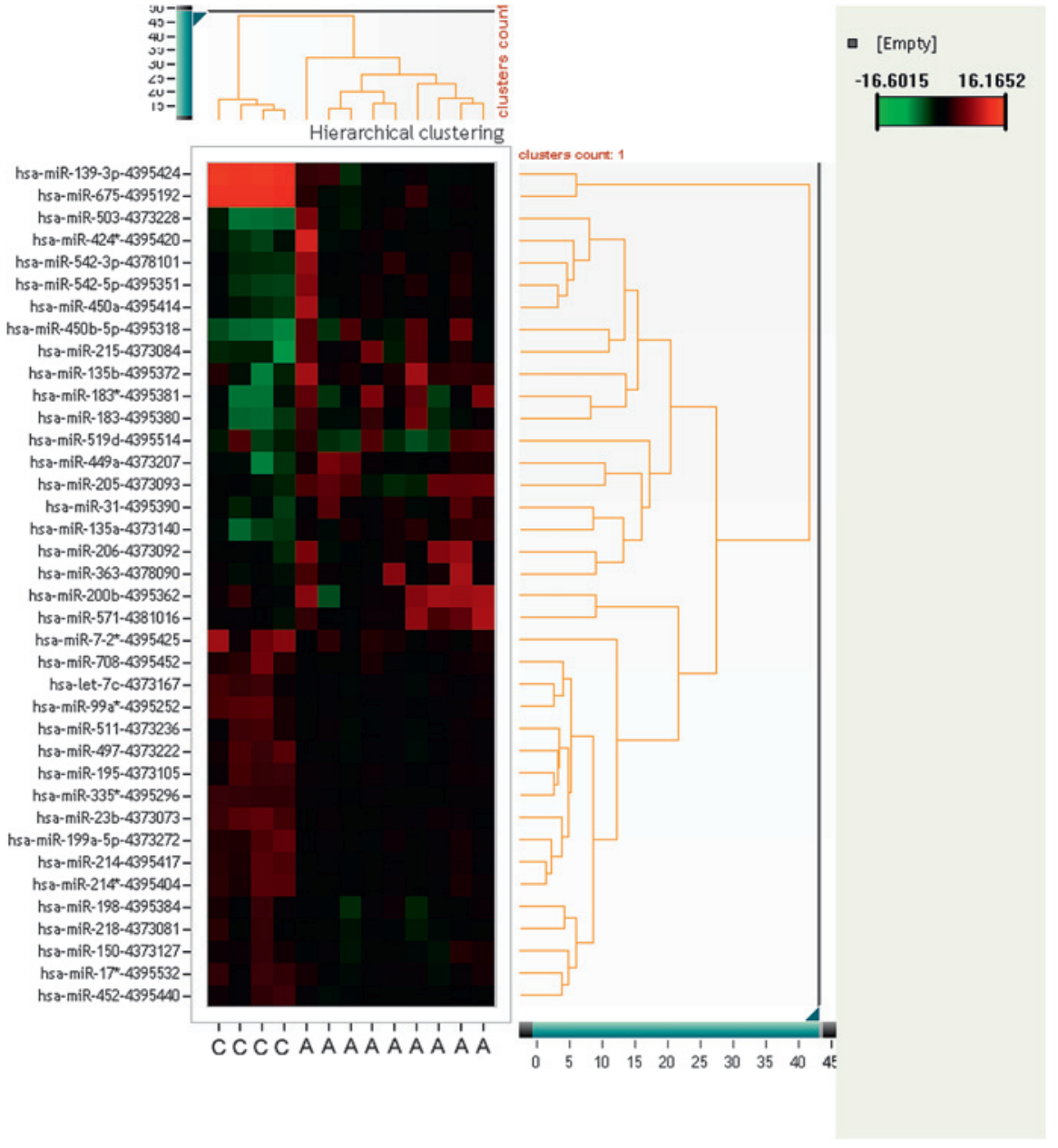

in normal adrenocortical tissue, ACA with Conn or Cushing syndrome, as well as ACCs and tissue from ACC metastases. In the groups of ACCs and ACAs, several miRNAs with differential expression were identified. Figure 1 shows a series of miRNAs identified as possibly useful for discriminating ACC from ACA.

\section{Normal adrenocortical tissue versus ACA associated with Cushing syndrome}

miRNA expression profiling showed 146 miRNAs to be significantly up-regulated and 17 miRNAs to be down-regulated in ACAs associated (online supplement).

\section{Normal adrenocortical tissue versus ACA associated with Conn syndrome}

miRNA expression profiling showed nine miRNAs to be significantly up-regulated and 47 miRNAs to be down-regulated (online supplement).
Normal adrenocortical tissue versus ACC

MiRNA expression profiling showed 74 miRNAs to be significantly up-regulated and 62 miRNAs to be down-regulated (online supplement).

Normal adrenocortical tissue versus tissue from ACC metastases miRNA expression profiling showed 140 miRNAs to be significantly up-regulated and 22 miRNAs to be down-regulated (online supplement)

\section{ACA (Conn syndrome) versus ACC}

miRNA expression profiling showed 38 miRNAs to be significantly up-regulated and 89 miRNAs to be down-regulated (online supplement).

\section{Adrenocortical adenoma (Cushing syndrome) versus adrenocortical} carcinoma

MiRNA expression profiling showed 159 miRNAs to be significantly up-regulated and 35 miRNAs to be down-regulated (online supplement).

Table 2 Expression levels of hsa-miR-139-3p-4395424, hsa-miR-675-4395192 and hsa-miR-335*-4395296 in normal, benign and malignant adrenocortical lesions

\begin{tabular}{|c|c|c|c|c|c|c|c|c|c|c|c|c|c|c|c|c|c|c|c|c|c|}
\hline \multirow{3}{*}{$\frac{\text { miRNA }}{\text { hsa-miR-139-3p-4395424 }}$} & & & & & & \multicolumn{9}{|c|}{ Adenomas $(n=9)$} & & & & & & & \\
\hline & \multicolumn{5}{|c|}{ Normal tissue $(n=5)$} & \multicolumn{4}{|c|}{ Conn } & \multicolumn{5}{|c|}{ Cushing } & \multicolumn{4}{|c|}{ Carcinomas $(n=4)$} & \multicolumn{3}{|c|}{ Metastasis $(n=3)$} \\
\hline & 7.0 & 7.6 & 7.3 & 7.3 & 6.4 & 8.1 & 6.0 & 6.7 & 7.1 & 6.8 & 5.6 & 8.0 & 6.3 & 4.3 & 9.7 & 10.8 & 9.7 & 10.6 & 9.8 & 11.3 & $7.3^{*}$ \\
\hline hsa-miR-675-4395192 & 6.7 & 5.9 & 6.8 & 5.8 & 5.8 & 8.8 & 6.0 & 7.8 & 6.4 & 6.3 & 5.8 & 5.0 & 7.1 & 4.3 & 10.8 & 11.6 & 10.1 & 10.5 & 10.5 & 10.2 & $7.5^{*}$ \\
\hline hsa-miR-335*-4395296 & 5.2 & 5.1 & 4.3 & 5.6 & 5.1 & 6.1 & 5.4 & 5.8 & 5.6 & 4.6 & 5.3 & 5.4 & 4.9 & 3.4 & 6.9 & 7.7 & 7.1 & 8.0 & 7.8 & 10.2 & $5.6^{*}$ \\
\hline
\end{tabular}

Data presented in $\triangle \mathrm{CT}$ of relative threshold cycles indicating fold changes over normal adrenal tissue. Low values indicate high levels of miRNA. Data derived from quantitative reverse transcriptase PCR validation of miRNAs.

${ }^{*}$ miRNA expression levels of this case was beyond the expected range. The amplification curves suggest an insufficient RNU48 control. However, data was not deleted from statistics. 
Table 3 Differential expression of hsa-miR-139-39, hsa-miR-675 and hsa-miR-335 in normal adrenocortical tissue, adrenocortical adenomas and adrenocortical carcinomas as well as metastasis

\begin{tabular}{lcc}
\hline miRNA & Fold change & p Value \\
\hline ACA compared to normal adrenocortical tissue; fold change normalised to normal \\
tissue & $1.34 \downarrow$ & \\
hsa-miR-139-3p & $1.10 \uparrow$ & 0.331 \\
hsa-miR-335 & $1.13 \uparrow$ & 0.772 \\
hsa-miR-675 & 0.778 \\
ACC compared to normal adrenocortical tissue; fold change normalised to normal \\
tissue \\
hsa-miR-139-3p & $8.49 \downarrow$ & $<0.001$ \\
hsa-miR-335 & $5.25 \downarrow$ & $<0.001$ \\
hsa-miR-675 & $23.25 \downarrow$ & $<0.001$ \\
ACC compared to ACA; fold change normalised to ACA & \\
hsa-miR-139-3p & $11.34 \downarrow$ & $<0.001$ \\
hsa-miR-335 & $4.85 \downarrow$ & $<0.001$ \\
hsa-miR-675 & $20.42 \downarrow$ & $<0.001$ \\
Metastasis compared to ACA; fold change normalised to adrenocortical adenoma \\
hsa-miR-139-3p & $6.09 \downarrow$ & 0.008 \\
hsa-miR-335 & $6.55 \downarrow$ & 0.008 \\
hsa-miR-675 & $8.12 \downarrow$ & 0.010 \\
\hline
\end{tabular}

List of key miRNAs with differential expression in normal adrenal tissue $(n=5)$, adrenocortical adenomas $(n=9)$, adrenocortical carcinomas $(n=4)$ and metastasis $(n=3)$. ACA, adrenocortical adenoma; ACC, adrenocortical carcinoma.

\section{Quantitative reverse transcriptase PCR validation of miRNAs}

Due to the large amount of significantly differentially expressed miRNAs, three key miRNAs were selected for further validation by quantitative reverse transcriptase PCR: miRNA hsa-miR-139-3p-4395424, hsa-miR-675-4395192 and hsa-miR$335^{*}-4395296$. High values of relative threshold cycles indicate low miRNA quantity, and data were normalised to RNU48 expression. Data from miRNA expression levels of one case with metastatic tissue was beyond the expected range. Although the amplification curve suggested an insufficient RNU48 control in this particular experiment, we did not delete this data from further statistical analysis.

\section{Expression levels of miR-675}

The lowest level of miR-675 was found in the group of ACCs (10.76, SD 0.63), followed by metastatic tissue (9.43, SD 16.4), ACAs associated with Conn syndrome (7.28, SD 1.31), normal adrenocortical tissue (0.622, SD 0.49), and ACAs associated with Cushing syndrome $(5.71,1.08)$. In the subgroup of ACCs and ACAs, miR-675 expression of ACCs was distinctly different from that of ACAs with a down-regulation of miR-675 in ACCs of up to 20.42-fold. Compared to normal adrenal tissue, we found down-regulation of miR-675 in ACCs up to 23.25-fold. Tables 2 and 3 present data on miR-675 expression levels. Figure 2 shows the distribution of expression levels among the different subgroups.

\section{Expression levels of miR-139-3p}

The lowest level of miR-139-3p was found in the group of ACCs (10.19, SD 0.56), followed by metastatic tissue (9.56, SD 1.92), normal adrenocortical tissue (7.10, SD 0.43), ACAs associated with Conn syndrome (6.98, SD 0.87), and ACAs associated with Cushing syndrome $(6.19,1.35)$. In the subgroup of ACCs and ACAs, miR-139-3p expression of ACCs was distinctly different from that of ACAs with a down-regulation of miR-139-3p in ACCs of up to 8.49-fold. Compared to normal adrenal tissue, we found down-regulation of miR-139-3p in ACCs up to 11.34-fold. Tables 2 and 3 present data on miR-139-3p expression levels. Figure 2 shows the distribution of expression levels among the different subgroups.
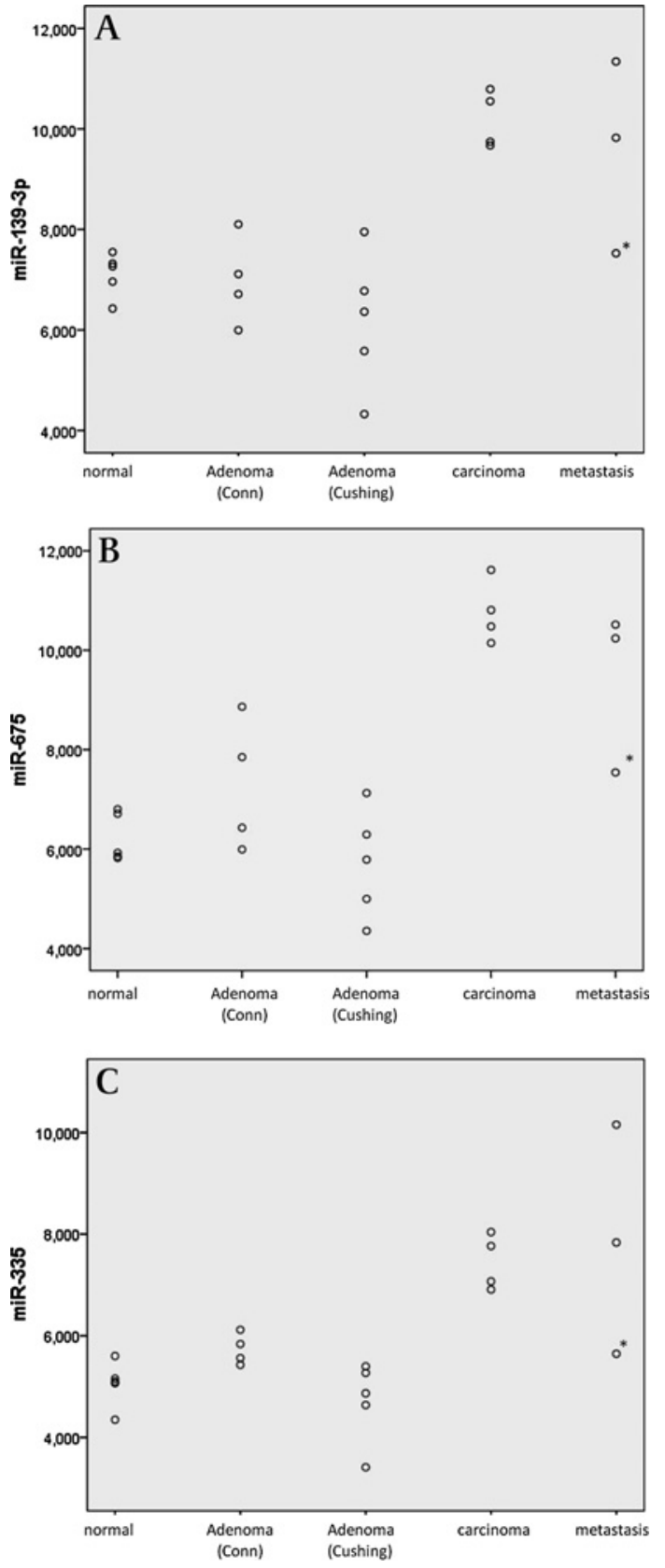

Figure 2 (A) Expression levels of hsa-miR-139-3p-4395424 in normal, benign and malignant adrenocortical lesions. Data derived from quantitative real-time PCR and presented in $\triangle \mathrm{CT}$ of relative threshold cycles indicating fold changes over normal adrenal tissue. Low values indicate high levels of miRNA. (B) Expression levels of hsa-miR-6754395192 in normal, benign and malignant adrenocortical lesions. Data derived from quantitative real-time PCR and presented in $\triangle \mathrm{CT}$ of relative threshold cycles indicating fold changes over normal adrenal tissue. Low values indicate high levels of miRNA. (C) Expression levels of hsa-miR$335^{*}-4395296$ in normal, benign and malignant adrenocortical lesions. Data derived from quantitative real-time PCR and presented in $\triangle \mathrm{CT}$ of relative threshold cycles indicating fold changes over normal adrenal tissue. Low values indicate high levels of miRNA.

\section{Expression levels of miR-335}

The lowest level of miR-335 was found in the group of metastatic tissue (7.87, SD 2.25), followed by ACCs (7.44, SD 0.54), ACAs associated with Conn syndrome (5.73, SD 0.30), normal adrenocortical tissue (5.05, SD 0.45), and ACAs associated with Cushing syndrome $(4.71,0.79)$. In the subgroup of ACCs and 
ACAs, miR-335 expression of ACCs was distinctly different from that of ACAs, with a down-regulation of miR-335 in ACCs of up to 4.85-fold. Compared to normal adrenal tissue, we found down-regulation of miR-335 in ACCs up to 5.25-fold. Tables 2 and 3 present data on miR-335 and miR-675 expression levels. Figure 2 shows the distribution of expression levels among the different subgroups.

\section{Validation cohort}

The three key miRNAs, hsa-miR-139-3p-4395424, hsa-miR-6754395192 and hsa-miR-335*-4395296, were selected for further validation by quantitative reverse transcriptase PCR in a validation cohort of 15 primary adrenocortical lesions, all scored according the Weiss score and one lung metastasis. Table 4 presents relevant clinicopathological data. Cases were sorted according to increasing Weiss scores. The cut-off values of the different miRNAs indicating a malignant behaviour were derived from the test cohort and are as followed: miRNA 335, $>6$; miRNA $139,>9$; miRNA $675,>8.8$.

\section{miRNA-335}

Regarding the expression levels of miRNA-335, all cases classified as benign with a Weiss score of 0 showed values below the cut-off of 6 . Among the lesions with uncertain biological behaviour (Weiss score 1-2), one case exhibited an expression level above the cut-off. Lesions with a Weiss score of 3 to 5 revealed in three of five cases increased expression levels above the cut-off value.

\section{miRNA-139-3p}

The expression levels of miRNA-139 in cases classified as benign (Weiss score $=0$ ) were lower than the cut-off value in five cases. One case exhibited increased expression levels. Among the five lesions with uncertain biological behaviour (Weiss score 1-2) the cut-off value was not reached. All lesions with a Weiss score of 3 to 5 lacked increased expression values. In one case data was not available.

\section{miRNA-675}

All adrenocortical tumours with a Weiss score of 0 showed low expression levels, not reaching above the cut-off value. One of the lesions with uncertain behaviour (Weiss score 1) exhibited values above the cut-off level. Of the five lesions with marked increased Weiss scores including the metastasis, two specimens showed expression levels above the cut off-level. Except for one case, all specimens showed increased expression levels nearly reaching the cut-off value.

\section{DISCUSSION}

The differential diagnosis of ACA from ACA is classically based on a set of clinical and pathomorphological parameters. However, taken alone, none of these parameters is sufficient to prove malignant behaviour. To overcome the problem, differing scoring systems have been developed which are without exception rather complicated, time consuming and difficult to reproduce. $^{20} 21$ Therefore novel markers allowing a more reliable separation of benign from malignant adrenocortical lesions are desirable. Molecular techniques such as miRNA analysis possess significant diagnostic potential. ${ }^{22}$

miRNA arrays can be used to differentiate different types of tissue such as non-neoplastic pancreatic tissue from human pancreatic adenocarcinoma ${ }^{23}$ or non-neoplastic prostatic tissue from prostatic adenocarcinoma. ${ }^{24}$ In addition, various studies have been successful in correlating miRNA expression levels with survival time in different cancer types, such as pancreatic carcinoma ${ }^{2425}$ and colorectal cancer. ${ }^{26}$ A previous study of our group found miRNA expression analysis to be helpful for the differential diagnosis of various types of polyps of the colorectum. $^{27}$ In endocrine pathology, miRNA analysis has been established as a useful tool in thyroid tumour pathology. Using specific sets of miRNAs, various benign and malignant tumour entities can be associated with characteristic up- or down-regulation patterns. ${ }^{5-10}$

Concerning adrenocortical pathology, a study on 22 ACAs and 33 ACCs using DNA array analysis managed to accurately classify benign and malignant tumours and offered novel prognostic information derived from gene expression patterns. ${ }^{11}$ Very recently, Soon et $a l^{9}$ published a study on 22 ACAs and 27 ACCs, suggesting the diagnostic and prognostic value of even single miRNAs. miRNA analysis thus seems to facilitate separation of adrenocortical neoplasm.

In this study we analysed 667 miRNAs in order to further strengthen the foundation for the miRNA based classification and differentiation of normal adrenocortical tissue, ACAs, ACCs and ACC metastasis. In a second step we validated expression data of three highly significantly altered single key miRNAs using real-time PCR. A third step was conducted with

Table 4 miRNA expression values in the validation cohort

\begin{tabular}{|c|c|c|c|c|c|c|c|}
\hline & Tumour size & Tumour weight & Weiss score & Metastasis & miR-335 cut-off $>6$ & miR-139-3p cut-off $>9$ & miR-675 cut-off $>8.8$ \\
\hline Case 1 & $2 \mathrm{~cm}$ & $\times$ & 0 & No & 5.399 & 8.247 & 4.462 \\
\hline Case 2 & $2 \mathrm{~cm}$ & $\times$ & 0 & No & 5.678 & 7.544 & 5.322 \\
\hline Case 3 & $3 \mathrm{~cm}$ & $7 \mathrm{~g}$ & 0 & No & 4.820 & 9.165 & 6.318 \\
\hline Case 4 & $3 \mathrm{~cm}$ & $x$ & 0 & No & 5.359 & 7.661 & 6.805 \\
\hline Case 5 & $3 \mathrm{~cm}$ & $\times$ & 0 & No & 3.131 & 4.928 & 6.046 \\
\hline Case 6 & $4.5 \mathrm{~cm}$ & $40 \mathrm{~g}$ & 0 & No & 4.532 & 7.131 & 5.653 \\
\hline Case 7 & $2.4 \mathrm{~cm}$ & $\times$ & 1 & No & 5.299 & 6.523 & 5.527 \\
\hline Case 8 & $3 \mathrm{~cm}$ & $34 \mathrm{~g}$ & 1 & No & 4.611 & 6.504 & 6.665 \\
\hline Case 9 & $5 \mathrm{~cm}$ & $\times$ & 1 & No & 4.796 & 7.997 & 9.326 \\
\hline Case 10 & $6 \mathrm{~cm}$ & $\times$ & 2 & No & 6.217 & 3.909 & 3.970 \\
\hline Case 11 & $9 \mathrm{~cm}$ & $180 \mathrm{~g}$ & 2 & No & 4.124 & 7.084 & 7.720 \\
\hline Case 12 & $4.5 \mathrm{~cm}$ & $\times$ & 3 & No & 5.959 & $\times$ & 3.727 \\
\hline Case 13 & $9.3 \mathrm{~cm}$ & $180 \mathrm{~g}$ & 4 & No & 7.683 & 5.242 & 8.813 \\
\hline Case 14 & $8 \mathrm{~cm}$ & $110 \mathrm{~g}$ & 5 & No & 7.321 & 6.386 & 8.749 \\
\hline Case 15 & $14 \mathrm{~cm}$ & $500 \mathrm{~g}$ & 5 & Yes (lung) & 5.319 & 7.011 & 9.023 \\
\hline Case 16 & $\times$ & $x$ & Metastasis & Yes (lung, liver) & 6.728 & 8.764 & 8.059 \\
\hline
\end{tabular}


Table 5 Potential targets for miRNA-675, miR-139-3p and miR-335

\begin{tabular}{|c|c|c|c|c|}
\hline & Ensemble gene ID & miTG Score & Precision & Gene description \\
\hline \multicolumn{5}{|l|}{ miR-139-3p } \\
\hline GLI4 & ENSG00000181638 & 12.00 & 0.83 & Zinc finger protein GLI4 (Krueppel-related zinc finger protein 4) \\
\hline ZFP41 & ENSG00000181638 & 12.00 & 0.83 & Zinc finger protein GLI4 (Krueppel-related zinc finger protein 4) \\
\hline C10orf39 & ENSG00000188385 & 9.00 & 0.4 & CDNA FLJ45017 fis \\
\hline MLC1 & ENSG00000100427 & 8.00 & 0.4 & Membrane protein MLC1 \\
\hline BARHL1 & ENSG00000125492 & 8.00 & 0.4 & BarH-like one homoeobox protein. \\
\hline GLI4 & ENSG00000181638 & 18.00 & 0.98 & Zinc finger protein GLI4 \\
\hline ZFP41 & ENSG00000181638 & 18.00 & 0.98 & Zinc finger protein GLI4 \\
\hline MGAM & ENSG00000179087 & 9.00 & 0.83 & Maltase-glucoamylase \\
\hline \multicolumn{5}{|l|}{ miR-335 } \\
\hline NEBL & ENSG00000078114 & 12.42 & 0.18 & Nebulette (actin-binding Z-disk protein) \\
\hline C8orf44 & ENSG00000213865 & 9.66 & 0.18 & Putative uncharacterised protein C8orf44 \\
\hline KPNA6 & ENSG00000025800 & 9.07 & 0.18 & Importin subunit $\alpha-7$ (karyopherin subunit $\alpha-6$ ). \\
\hline TNFAIP1 & ENSG00000109079 & 9.00 & 0.18 & BTB/POZ domain-containing protein TNFAIP1 (tumour necrosis factor) \\
\hline ONECUT2 & ENSG00000119547 & 9.00 & 0.18 & One cut domain family member 2 \\
\hline UNC5D & ENSG00000119547 & 9.00 & 0.18 & One cut domain family member 2 (transcription factor ONECUT-2) (OC-2). \\
\hline UNC5D & ENSG00000156687 & 9.00 & 0.18 & Netrin receptor UNC5D precursor (Unc-5 homologue D) (Unc-5 homologue 4). \\
\hline MMAA & ENSG00000151611 & 9.00 & 0.18 & Methylmalonic aciduria type A protein \\
\hline
\end{tabular}

Ensemble gene ID: provides a link to the Ensembl page of the gene, which contains information on sequence, alignments, protein products and links to much more information. Total miTG score: the final prediction score. A higher miTG score corresponds to a higher possibility of being a real target. A non-strict (default) threshold is set at score 7.3 and a strict threshold at score 19 .

a validation cohort in order to confirm the results. The present study was performed with paraffin-embedded tissue which was recently shown to be suitable for miRNA arrays. ${ }^{28}$ Our study reveals up to 146 statistically significantly differentially expressed miRNAs in the various subgroups analysed. Thus this study confirms the power of molecular profiling as a novel diagnostic tool for the diagnosis of adrenocortical tumours. Our results show that TLDA based assessment can assist in separating malignant from benign lesions. Due to the huge amount of differentially expressed miRNAs, we focused on three single key miRNAs with the most significantly differential expression: miRNA 139-3p, miRNA 675 and miRNA 335. Each of these miRNAs allowed separation of ACCs from ACAs in the test cohort. We observed distinctly different miRNA expression levels, with up to 23.25-fold down-regulation of miR-675 in ACC compared to normal adrenocortical tissue and up to 20.42fold down-regulation of miR-675 in ACC compared to ACA. Our results are in accordance with those of Soon et al, ${ }^{16}$ who found 23 miRNAs to be differentially expressed between ACA and ACC. Among these are miRNA 335 and miRNA195, which our study also identified as diagnostically valuable miRNAs.

We were aware that the test cohort needed further confirmation using a validation cohort. The validation cohort consisted of adrenocortical tumours with known Weiss score, including six benign tumours without any histopathological criteria proposed by Weiss (score 0 ). In fact, none of the three selected miRNAs correlated directly with the Weiss score. However, miRNA-335 and miRNA-675 show a potential diagnostic value in predicting the biological behaviour of adrenocortical tumours. In the subgroup containing cases with a marked increased Weiss score (score $\geq 3$ ) or histologically proven metastasis, miRNA-335 and miRNA-675 levels were up-regulated in three of five and in three of five adrenocortical tumours, respectively. In contrast, miRNA-139 did not prove to be a useful tool for identifying the potential biological behaviour of adrenocortical lesions. MiRNA-139 was up-regulated in several ACAs (Weiss score $=0$ ) of the validation cohort and lacked up-regulation in cases with malignant potential (Weiss score $\geq 3$ ). Using a cut-off value derived from the test cohort, analysis of miR-335 expression levels identified correctly three of five cases with highly probable malignant course. Moreover miR-335 and 675 levels were markedly increased in two cases, with a Weiss score of 1 and 2, respectively. It is tempting to speculate that these cases may exhibit a malignant course. Unfortunately no long term follow-up data exist for these two patients. Especially in the grey zone of tumours with indeterminate malignant potential (Weiss score 1-2), miRNA levels show wide variations, indicating different genetic background alterations in these tumours.

Furthermore, this study confirms that miRNAs extracted from formalin-fixed and paraffin-embedded material can be successfully and reliably applied in routine diagnostic pathology.

There seems to be a large number of significantly expressed miRNAs in adrenal tumour pathology, depending on the nature

\section{Take-home messages}

- MicroRNAs (miRNAs) possess a tremendous potential as diagnostic biomarkers in surgical pathology.

- Up to 159 miRNAs were up-regulated and up to 89 were down-regulated in adrenocortical carcinomas (ACCs) compared to adrenocortical adenomas (ACAs).

- miR-335 and miR-675 expression levels helped to distinguish ACAs from ACCs and may help in identifying adrenocortical tumours with malignant behaviour. 
of the lesion as well as the underlying endocrine pathobiology resulting in either Conn or Cushing syndrome. The DIANAmicro T algorithm was used to predict possible target genes of the identified key miRNAs (table 5).

Interestingly, miRNA-675 and miRNA-139-3p share a common target gene (GLI4). However, this basic study was not designed to analyse possible functional effects of single miRNAs. Further experiments are in progress to elucidate the association of miRNA regulation/deregulation with already identified or putative target genes. We are aware that at this point of time little is known about the potential functional relevance of the identified miRNA in adrenocortical tumours.

In conclusion, this study provides a rich source of information about miRNAs that are differentially expressed in normal, benign and malignant adrenocortical tumours. In contrast to the study of Soon et $a l^{16}$ we found a much larger number of miRNAs to be significantly differentiated between ACA and ACC. However, due to overlapping miRNA expression values in the validation cohort, a single miRNA value is not capable of clearly separating benign from malignant adrenocortical lesions. Nevertheless, using proposed cut-off values, expression analysis of miR-675 and miR-335 seems to offer highly valuable information about the potential subsequent malignant behaviour of lesions with indeterminate malignancy.

Funding This work has been supported by the local research fund (IFORES) of the University of Essen.

Competing interests None.

Provenance and peer review Not commissioned; externally peer reviewed.

\section{REFERENCES}

1. Lee RC, Feinbaum RL, Ambros V. The C. elegans heterochronic gene lin-4 encodes small RNAs with antisense complementarity to lin-14. Cell 1993:75:843-54.

2. Wightman B, Ha I, Ruvkun G. Posttranscriptional regulation of the heterochronic gene lin-14 by lin-4 mediates temporal pattern formation in C. elegans. Cell 1993; 75:855-62.

3. Bartel DP. MicroRNAs: genomics, biogenesis, mechanism, and function. Cell 2004;116:281-97.

4. Vandenboom li TG, Li Y Philip PA, et al MicroRNA and Cancer: Tiny molecules with Major Implications. Curr Genomics 2008;9:97-109.

5. Pallante $\mathbf{P}$, Visone R, Ferracin $\mathrm{M}$, et al. MicroRNA deregulation in human thyroid papillary carcinomas. Endocr Relat Cancer 2006;13:497-508.

6. Pallante $\mathbf{P}$, Visone R, Croce CM, et al. Deregulation of microRNA expression in follicular cell-derived human thyroid carcinomas. Endocr Relat Cancer 2010;17: F91-104.
7. Nikiforova MN, Tseng GC, Steward D, et al. MicroRNA expression profiling of thyroid tumors: biological significance and diagnostic utility. J Clin Endocrinol Metab 2008;93:1600-8.

8. Sheu SY, Grabellus F, Schwertheim S, et al. Lack of correlation between BRAF V600E mutational status and the expression profile of a distinct set of miRNAs in papillary thyroid carcinoma. Horm Metab Res 2009:41:482-7.

9. Schwertheim S, Sheu SY, Worm K, et al. Analysis of deregulated miRNAs is helpful to distinguish poorly differentiated thyroid carcinoma from papillary thyroid carcinoma. Horm Metab Res 2009:41:475-81.

10. Sheu SY, Grabellus F, Schwertheim S, et al. Differential miRNA expression profiles in variants of papillary thyroid carcinoma and encapsulated follicular thyroid tumours. Br J Cancer 2010:102:376-82

11. Giordano TJ, Kuick R, Else $T$, et al. Molecular classification and prognostication of adrenocortical tumors by transcriptome profiling. Clin Cancer Res 2009;15:668-76.

12. Aubert S, Wacrenier A, Leroy $\mathrm{X}$, et al. Weiss system revisited: a clinicopathologic and immunohistochemical study of 49 adrenocortical tumors. Am J Surg Pathol 2002:26:1612-19.

13. DeLellis RA, Lloyd RV, Heitz PU. World Health Organization Classification of Tumors, Pathology and Genetics. Tumors of Endocrine Organs. Lyon, France: IARC Press, 2004.

14. van SH, Schaberg A, Smeenk D, et al. Morphologic characteristics of benign and malignant adrenocortical tumors. Cancer 1985;55:766-73.

15. Weiss LM, Medeiros LJ, Vickery AL Jr. Pathologic features of prognostic significance in adrenocortical carcinoma. Am J Surg Pathol 1989;13:202-6.

16. Soon PS, Tacon LJ, Gill AJ, et al. miR-195 and miR-483-5p Identified as Predictors of Poor Prognosis in Adrenocortical Cancer. Clin Cancer Res 2009;15:7684-92.

17. Maragkakis $\mathbf{M}$, Reczko M, Simossis VA, et al. DIANA-microT web server: elucidating microRNA functions through target prediction. Nucleic Acids Res 2009;37:W273-6. (Web Server issue).

18. Selbach M, Schwanhausser B, Thierfelder N, et al. Widespread changes in protein synthesis induced by microRNAs. Nature 2008;455:58-63.

19. Schmittgen TD, Livak KJ. Analyzing real-time PCR data by the comparative C(T) method. Nat Protoc 2008:3:1101-8.

20. Aiba M, Fujibayashi M. Histopathological diagnosis and prognostic factors in adrenocortical carcinoma. Endocr Pathol 2005;16:13-22.

21. Wieneke JA, Thompson LD, Heffess CS. Adrenal cortical neoplasms in the pediatric population: a clinicopathologic and immunophenotypic analysis of 83 patients. $\mathrm{Am} J$ Surg Pathol 2003;27:867-81.

22. Giordano TJ. Molecular pathology of adrenal cortical tumors: separating adenomas from carcinomas. Endocr Pathol 2006;17:355-63.

23. Lee EJ, Gusev Y, Jiang J, et al. Expression profiling identifies microRNA signature in pancreatic cancer. Int J Cancer 2007;120:1046-54.

24. Porkka KP, Pfeiffer MJ, Waltering KK, et al. MicroRNA expression profiling in prostate cancer. Cancer Res 2007:67:6130-5

25. Bloomston $\mathbf{M}$, Frankel WL, Petrocca $F$, et al. MicroRNA expression patterns to differentiate pancreatic adenocarcinoma from normal pancreas and chronic pancreatitis. JAMA 2007;297:1901-8.

26. Schetter AJ, Leung SY, Sohn JJ, et al. MicroRNA expression profiles associated with prognosis and therapeutic outcome in colon adenocarcinoma. JAMA 2008:299:425-36.

27. Schmitz KJ, Hey S, Schinwald A, et al. Differential expression of microRNA 181b and microRNA 21 in hyperplastic polyps and sessile serrated adenomas of the colon Virchows Arch 2009:455:49-54.

28. Zhang X, Chen J, Radcliffe $\mathrm{T}$, et al. An array-based analysis of microRNA expression comparing matched frozen and formalin-fixed paraffin-embedded human tissue samples. J Mol Diagn 2008;10:513-19. 